http://jmscr.igmpublication.org/home/ ISSN (e)-2347-176x ISSN (p) 2455-0450 crossref DOI: https://dx.doi.org/10.18535/jmscr/v8i3.29

\title{
Hypoglycemia: An Acute Coronary Syndrome Mimic
}

\author{
Authors \\ Vishal Singh $^{1^{*}}$, Satish Chandra Mishra ${ }^{2}$, Binod B Raut ${ }^{3}$ \\ ${ }^{1}$ Dept of Nephrology, 7 Air Force Hospital, Kanpur Cantonment, UP, India \\ ${ }^{2}$ Dept of Interventional Cardiology, Military Hospital-CTC, Pune, Maharashtra, India \\ ${ }^{3}$ Dept of Medicine, 7 Air Force Hospital, Kanpur Cantonment, UP, India \\ *Corresponding Author \\ Vishal Singh
}

\begin{abstract}
Background: Type-2 diabetes mellitus (T2DM) is a major risk factor for cardiovascular events. A good glycemic control, while reducing the risk of cardiovascular events, increases the risk of hypoglycemia. These hypoglycaemic events, at times, may precipitate reversible electrocardiographic (ECG) changes, causing a diagnostic dilemma.

Case Description: A 55 years old lady with long-standing T2DM and diabetic kidney disease presented with anginal chest pain, ST-segment depression involving the anterior chest leads, elevated cardiac biomarkers, and neuroglycopenic hypoglycemic episode. The coronary angiogram was normal. The ECG normalized spontaneously following the correction of blood sugars.

Conclusions: Severe acute hypoglycemic episode, may at times, present with reversible ECG changes mimicking myocardial ischemia. In such circumstances, it is prudent to repeat the ECG after blood sugar correction, before instituting invasive interventions.
\end{abstract}

Keywords: Acute hypoglycaemia; Acute Coronary Syndrome; Electrocardiographic Changes.

\section{Introduction}

The epidemic of type 2 diabetes mellitus (T2DM) poses a major challenge to the health care system worldwide. Hyperglycemia induces widespread tissue damage and is a major risk factor for cardiovascular diseases ${ }^{[1]}$. Amongst the many interventions directed towards lowering cardiovascular risk, one key intervention is good glycemic control. A good glycemic control while reducing the risk for cardiovascular events, increases the risk for hypoglycemia.

When a patient with T2DM in the appropriate clinical setting presents with an electrocardiographic (ECG) change, more often than not, it is attributed to underlying coronary artery disease. It is pertinent to note that hypoglycemia, by itself, can induce ECG changes. Occasionally, these ECG changes, pose a diagnostic and therapeutic dilemma. We present a case of T2DM, where a patient with an acute neuroglycopenic hypoglycemic episode presented as an acute coronary syndrome mimic.

\section{Case Report}

A 55 years old, post-menopausal lady, was diagnosed to have T2DM, hypertension, and dyslipidemia 12 years back. Two years back, she was noted to have obesity, diabetic kidney disease, non-proliferative diabetic retinopathy, and 
peripheral neuropathy. The serum creatinine was 1.9 milligrams/deciliter (estimated glomerular filtration rate- $29.2 \mathrm{ml} / \mathrm{min} / 1.73 \mathrm{~m}^{2}$ ) and the albuminuria was 1.2 grams per day. Her medications included sulfonylurea, biguanides, angiotensin receptor blockers and statins. The blood pressures and blood sugars were well controlled. Her exercise tolerance was good.

She was brought by her relatives to the emergency department in an unconscious state. She had been fasting for the past 24 hours for religious reasons. Two hours prior to the admission she complained of precordial chest pain, palpitations, shortness of breath and profuse generalized sweating. This was followed an hour later by the loss of consciousness. At admission, she was comatose with a Glasgow coma score of E1V1M1. The pulse was 130 per minute; blood pressure was 90/60 millimeters of mercury, and the respiratory rate was 26 per minute. The oxygen saturation at room air was $82 \%$. She had leg edema and crepitations involving the dependent part of the lungs. The neurological examination did not show any lateralization. The ECG showed a horizontal to down-sloping STsegment depression involving the precordial leads, V1 to V3 (Figure 1). The cardiac-specific Troponin$\mathrm{T}$ was elevated. The blood sugar was 24 milligrams per deciliter, and the serum creatinine was 2.3 milligrams per deciliter. The other laboratory parameters were normal.

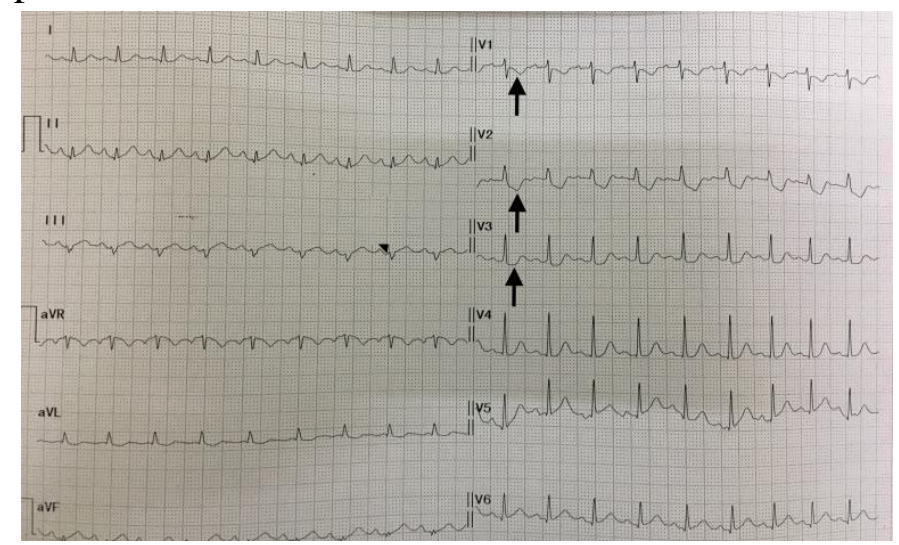

Fig. 1 ECG at presentation showing down-sloping ST segment depression involving the precordial leads V1 to V3 (arrow).

She was managed symptomatically in the emergency department with intravenous dextrose, fluid resuscitation, diuretics, and supplemental oxygen. Her sensorium rapidly improved and she became fully conscious within an hour. In view of her presenting symptoms, commensurate ECG changes and elevated cardiac biomarkers on the background of multiple cardiovascular risk factors, she was taken up for an urgent coronary angiography. The coronary angiogram showed normal epicardial blood vessels (Figure 2). A repeat ECG, done 3 hours following the hospitalization, showed spontaneous regression of ST-segment depression (Figure 3). The subsequent course in the hospital was uneventful. Her medications for T2DM were optimized and since then, she is asymptomatic. An exercise stress test, done later showed good effort tolerance with no evidence of reversible myocardial ischemia.

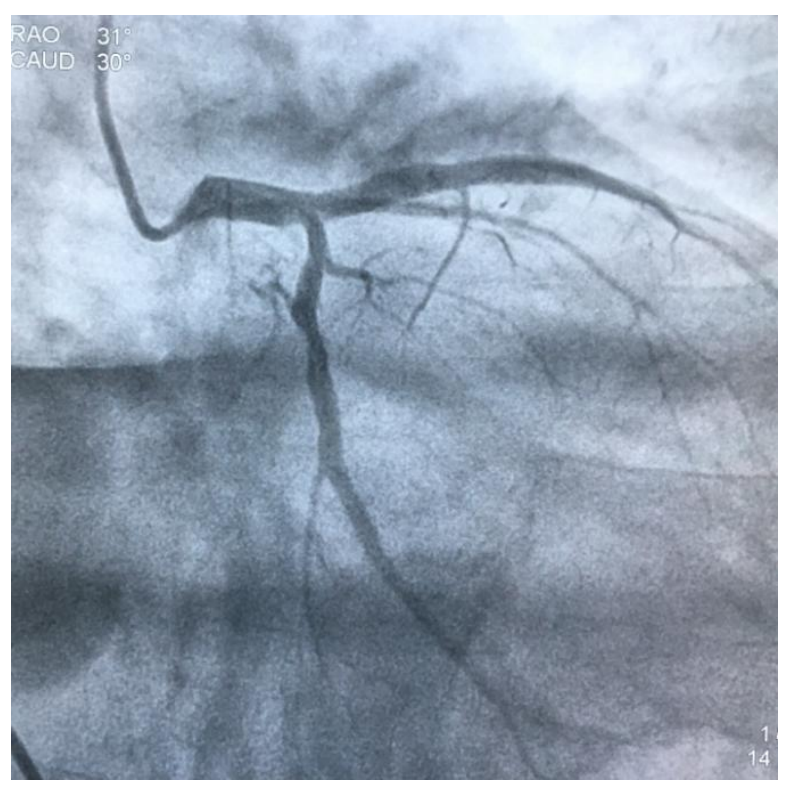

Fig. 2 Coronary angiogram showing normal epicardial coronaries

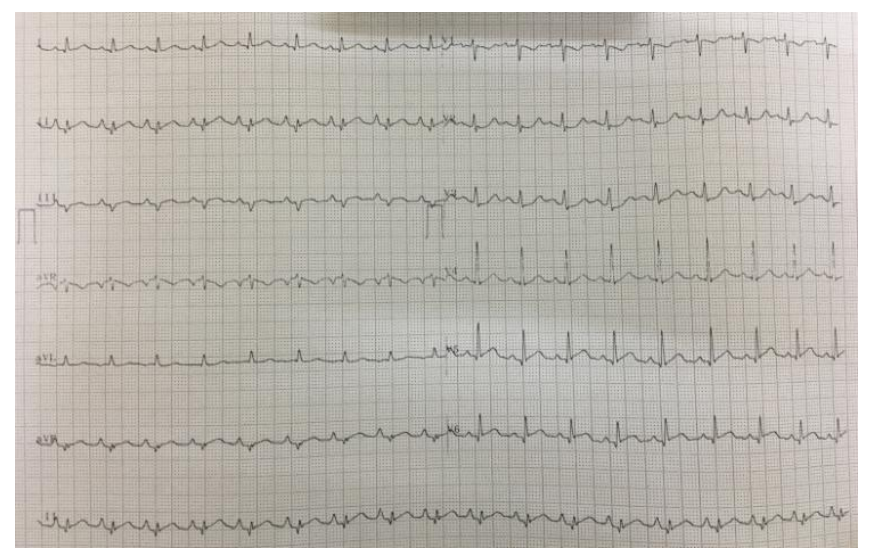

Fig. 3 Follow up ECG showing spontaneous regression of the ST segment depression. 


\section{Discussion}

Diabetes mellitus is a major risk factor for cardiovascular disease ${ }^{[1]}$. The presence of diabetes doubles the risk of cardiovascular events, and a vast majority die prematurely from myocardial infarction and stroke. Optimization of blood sugars, blood pressures, and lipids, along with lifestyle modification, forms the cornerstone of treatment. A good glycemic control while reduces the risk of cardiovascular events, increases the risk of hypoglycemia. When compared with standard therapy, the use of intensive therapy to target normal glycated hemoglobin levels increases mortality without reducing the incidence of major cardiovascular events ${ }^{[2,3]}$. There is thus a need to individualize the glycemic control goal. Renal dysfunction is seen in around one-third of diabetics, and in these, the cardiac biomarkers including troponins may be chronically elevated.

When a patient with T2DM presents with anginal chest pain along with commensurate ECG changes and elevated cardiac biomarkers, the commonest differential remains a coronary artery disease. At times, the explanation for such a presentation may be very different. Hypoglycemia in itself is known to induce ECG changes. The postulated mechanism includes the direct effect of hypoglycemia on the myocardium and its conducting system and secondary to the autonomic response. Acute hypoglycemia provokes autonomic response via the sympatho-adrenal system resulting in widespread hemodynamic changes. These include, increase in heart rate, systolic blood pressure, myocardial contractility, and stroke volume; and a fall in the peripheral vascular resistance ${ }^{[4]}$. These compensatory changes are aimed towards increasing the production and tissue-availability of glucose. Hypoglycemia can directly affect the myocardial cells. Various studies have demonstrated that insulin-induced hypoglycemia affects the repolarization of cardiac myocytes in both diabetics as well as non-diabetic individuals ${ }^{[5,6]}$. This altered repolarization manifests as a reduction in the amplitude of $\mathrm{T}$ waves, which flattens and lengthens. The heart rate corrected QT interval (QTc) is prolonged, thus increasing the susceptibility to arrhythmias and sudden cardiac death ${ }^{[7]}$. Some atypical ECG changes have also been described in association with hypoglycemia. In an unusual presentation, the author described a patient of T2DM with acute hypoglycemic episodes presenting with neuroglycopenic symptoms and an ECG with no discernable electrical activity. The ECG reverted to normal sinus rhythm following the correction of blood sugars ${ }^{[8]}$. Occasional case reports of angina and acute coronary syndrome precipitated by hypoglycemia have also been described $^{[9,10]}$.

Our patient differed significantly from the existing sparse case reports. She was initially thought to have an occult coronary disease, with myocardial ischemia being precipitated by a demand-supply mismatch induced by the sympathetic response. In the hindsight, it was realized that the initial assumption was wrong and an alternative explanation existed to explain the whole sequence of events.

In hypoglycemia, the adrenergic response usually precedes the neuroglycopenic episode. Her initial symptoms including palpitations, dyspnea, and diaphoresis represented an initial adrenergic response. The fluid overload and the false-positive cardiac biomarkers were secondary to acute on chronic kidney disease. The reason for the ECG changes remained an enigma. In earlier case reports, myocardial ischemia was provoked by the stress induced by the autonomic response on the background of occult coronary artery disease. The same was not true for our patient; she had normal coronaries, and the observed ECG changes rapidly normalized following the correction of hypoglycemia, suggesting a cause-effect relationship. To explain the observed ST changes on ECG, we propose the following hypothesis. Hypoglycemia, like hypoxia, leads to depletion of intracellular adenosine triphosphate (ATP). The autonomic response to hypoglycemia further worsens this demand-supply mismatch. This depleted myocardial ATP reserve leads to diminished activity of $\mathrm{Na}^{+} \mathrm{K}^{+}$ATPase pump which 
is responsible for membrane hyperpolarization. A decrease in the activity of the $\mathrm{Na}^{+} \mathrm{K}^{+}$ATPase pump would have led to membrane depolarization and the transient ST-segment changes observed were the result of an injury current flowing from the depolarized region to the normal region. Following the treatment, once the hypoglycemic milieu was corrected, volume status normalized and the hypoxia improved, the ECG spontaneously normalized.

We believe, this is the first reported case of hypoglycemia induced reversible ST-segment depression, where an underlying coronary artery disease has been conclusively ruled out. Further, based on the temporal profile, we were able to demonstrate the cause-effect relationship.

\section{Conclusions}

Acute hypoglycemia has a myriad presentation. At times, it may provoke transient, reversible ECG changes, causing a diagnostic dilemma. In such cases, it is prudent to repeat an ECG after correction of the blood sugars, before instituting invasive therapeutic intervention.

\section{References}

1. Morrish NJ, Wang SL, Stevens LK, Fuller JH, Keen H. Mortality and causes of death in the WHO Multinational Study of Vascular Disease in Diabetes. Diabetologia 2001;44 Suppl 2:S14-21.

2. ACCORD study group. Effects of intensive glucose lowering in type 2 diabetes. $\mathrm{N}$ Engl $\mathbf{J}$ Med 2008; 358:2545-59.

3. Duckworth W, Abraira C, Moritz T, Redad D, Emmanuele M, Reaven PD, et al. Glucose control and vascular complications in veterans with type 2 diabetes. N Engl J Med 2009; 360:129-39.

4. Wright RJ, Frier BM. Vascular disease and diabetes: is hypoglycaemia an aggravating factor? Diabetes Metab Res Rev 2008;24: 353-363.
5. J. Meinhold, T. Heise, K. Rave, and L. Heinemann, "Electrocardiographic changes during insulin-induced hypoglycemia in healthy subjects," Hormone and Metabolic Research, vol. 30, no. 11, pp. 694-697, 1998.

6. M. L. Koivikko, M. Karsikas, P. I. Salmela et al., "Effects of controlled hypoglycaemia on cardiac repolarisation in patients with type 1 diabetes," Diabetologia, vol. 51, no. 3, pp. 426-435, 2008.

7. Koivikko ML, Karsikas M, Salmela PI, et al. Effects of controlled hypoglycaemia on cardiac repolarisation in patients with type 1 diabetes. Diabetologia 2008; 51: 426-435.

8. Gupta AK. ECG in Hypoglycemia: Mimic Isoelectric ECG. JAPI 2011;59: 269-70.

9. Graveling AJ, Frier BM. Does hypoglycaemia cause cardiovascular events? Br J Diabetes Vasc Dis 2010; 10:5-13.

10. Kamijo Y, Soma K, Aoyama N, Fukuda N, Ohwada T. Myocardial infarction with acute insulin poisoning: A case report. Angiology 2000; 51:689-93. 